\title{
DIFFERENTIAL EXPRESSION OF NEUTROPHIL ADHESION MOLECULES DURING CORONARY ARTERY SURGERY WITH CARDIOPULMONARY BYPASS
}

\author{
Marcus K. Ilton, FRACPa,b \\ Paul E. Langton, FRACP, \\ Marcia L. Taylor, $\mathrm{PhD}^{\mathrm{a}}$ \\ Neil L. A. Misso, $\mathrm{PhD}^{\mathrm{a}}$ \\ Mark Newman, DS, FRACS \\ Philip J. Thompson, FRACP ${ }^{\mathrm{a}}$ \\ Joseph Hung, FRACPa,b
}

Background: Activation of neutrophil adhesion molecules and subsequent neutrophil adhesion to vascular endothelium are key events initiating inflammatory organ dysfunction after cardiopulmonary bypass and ischemic reperfusion. Objectives: We sought to characterize neutrophil integrin CD11b and L-selectin activation associated with coronary artery bypass graft surgery and to determine whether neutrophil activation contributes to their sequestration on postbypass reperfusion. Methods: Twenty patients undergoing routine coronary artery bypass were studied. Heparinized whole blood was simultaneously sampled from a central venous line, aorta, coronary sinus, and right and left atrium before, during, and up to 20 minutes after cardiopulmonary bypass. Neutrophil counts were obtained, and neutrophil CD11b and L-selectin expression was determined by flow cytometric analysis in whole blood. Results: CD11b expression on circulating neutrophils increased during cardiopulmonary bypass, peaking at $\mathbf{1 4 5 \%}$ of baseline level after release of the aortic clamp and then declined by 20 minutes after bypass (analysis of variance, $P=.003$ ). No change in neutrophil $L$-selectin expression was observed during cardiopulmonary bypass. Neutrophils responded to ex vivo stimulation by $\mathrm{C5a}$ and leukotriene $\mathrm{B}_{4}$ during cardiopulmonary bypass but not at 24 hours after the operation. After reperfusion, neutrophil loss, but not local activation, was demonstrated in the coronary and pulmonary circulations. Conclusions: Upregulated CD11b expression on neutrophils is likely to contribute to neutrophil sequestration in the heart and lungs after bypass, but neutrophil activation may be limited by their reduced responsiveness to agonist stimulation. CD11b represents a potential therapeutic target for diminishing inflammation after cardiac operations. (J Thorac Cardiovasc Surg 1999;118: 930-7) $\mathrm{c}$ ardiopulmonary bypass (CPB) is associated with systemic inflammation that can lead to generalized vascular and organ dysfunction described as the post-

From the Department of Medicine, ${ }^{\text {a }}$ University of Western Australia, the Department of Cardiovascular Medicine, b Sir Charles Gairdner Hospital (M.K.I., P.E.L., J.H.), and the Department of Cardiothoracic Surgery, ${ }^{c}$ Sir Charles Gairdner Hospital, Perth, Western Australia.

Supported by grants from the R. F. Shaw Foundation; Sir Charles Gairdner Hospital, Perth, Western Australia; and the Raine Medical Research Foundation, the University of Western Australia.

Received for publication April 19, 1999; revisions requested July 12, 1999; revisions received Aug 4, 1999; accepted for publication Aug 11, 1999.

Address for reprints: Joseph Hung, FRACP, Associate Professor, University Department of Medicine, Sir Charles Gairdner Hospital, Nedlands, WA 6009 Australia.

Copyright (C) 1999 by Mosby, Inc.

0022-5223/99 $\$ 8.00+0 \quad \mathbf{1 2 / 1 / 1 0 2 0 8 8}$ perfusion syndrome. ${ }^{1}$ Activation of neutrophils during $\mathrm{CPB}$, their adhesion to endothelial cells, and adhesiondependent release of cytotoxic products are largely responsible for mediating the inflammatory damage. ${ }^{2,3}$ Experimental interventions that suppress neutrophil activation, deplete neutrophils, or block their adhesion to endothelial cells have been shown to reduce the tissue injury associated with CPB and ischemia-reperfusion. ${ }^{4-7}$ A number of soluble mediators are known to activate neutrophils during $\mathrm{CPB}$ and ischemia-reperfusion, including activated complement anaphylatoxins $\mathrm{C} 3 \mathrm{a}$ and $\mathrm{C} 5 \mathrm{a},{ }^{8,9}$ tumor necrosis factor $\alpha$, interleukin (IL) 6 and IL-8, and leukotriene (LT) $\mathrm{B}_{4} \cdot{ }^{10-12}$ The cellular functions and interactions of leukocytes with the vascular endothelium are dependent on expression of surface cell adhesion receptors. ${ }^{2,13}$ L-selectin, which is constitutively expressed on nonactivated leukocytes, is responsible for 
leukocyte rolling and margination and is rapidly shed on chemotactic stimulation. Subsequent firm adhesion to activated endothelium is mediated by the upregulation of the $\beta_{2}$-integrin complex, particularly CD11b/CD18 (Mac-1). Thus downregulation (loss) of L-selectin and upregulation of CD11b are indicators of neutrophil activation and likely vascular transmigration. Previous studies have found an increased $\beta_{2}$-integrin expression on circulating blood neutrophils during $\mathrm{CPB},{ }^{14-16}$ but the extent to which ischemic reperfusion of the heart and lungs compared with a general CPB-induced inflammation contributes to the neutrophil activation has not been specifically studied. Whether L-selectin expression on neutrophils is also downregulated during human CPB is controversial because a decrease in expression has been found in some studies ${ }^{17,18}$ but not others. ${ }^{15,19,20}$ It is also important to assess whether neutrophil responsiveness to humoral stimulation can be downregulated after CPB because this may represent a significant in vivo mechanism to limit neutrophil participation in the inflammatory process.

Thus the aims of the present investigation in patients undergoing elective coronary artery bypass graft (CABG) were (1) to characterize the profile of activation and expression of surface adhesion molecules, CD11b and L-selectin, on circulating neutrophils during $\mathrm{CPB}$ and after organ reperfusion; (2) to measure changes in neutrophil responsiveness to ex vivo agonist stimulation during and after CPB; and (3) to determine the presence of neutrophil activation along with neutrophil sequestration in the heart and lungs after ischemic reperfusion.

\section{Methods}

Reagents. FACS lysis solution and the following flourophore-labeled monoclonal antibodies were purchased from Becton Dickinson-Pharmingen (Rutherford, NJ): mouse isotype (negative control) IgG1 fluorescein isothiocyanate (FITC)-IgG2 $\alpha$ phycoerythrin (PE); combined FITC-antiCD45:PE-anti-CD14 leukocyte typing mix; and FITC-antiL-selectin and PE-anti CD11b. Complement C5a was generated by in vitro zymosan activation of fresh human serum (zymosan $15 \mathrm{mg} / \mathrm{mL}$ serum), and C5a serum supernatant was stored at $-70^{\circ} \mathrm{C}$ until the day of use. $\mathrm{LTB}_{4}$ was purchased from Sapphire Bioscience (Alexandria, NSW, Australia) and stored at $-20^{\circ} \mathrm{C}$.

Subjects. Twenty patients (18 men and 2 women; mean age, 61 years; age range, 44-84 years) with stable angina and left ventricular ejection fraction of greater than $40 \%$ undergoing elective $\mathrm{CABG}$, including a graft to the left anterior descending artery, participated in the study. Their clinical characteristics are summarized in Table I. The profile of neutrophil activation and neutrophil sequestration during and after surgery was studied in an initial series of 12 patients. Ex
Table I. Characteristics of the study patients $(n=20)$ and $C A B G$

\begin{tabular}{ll}
\hline Age, y (mean [range]) & $61(44-84)$ \\
Sex (M/F) & $18 / 2$ \\
Degree of coronary disease (1/2/3-vessel stenosis) & $2 / 3 / 15$ \\
No. of grafts (median [range]) & $3(1-5)$ \\
CPB duration, minutes & $70 \pm 24$ \\
Aortic crossclamping (cardiac ischemia) duration, & $40 \pm 19$ \\
$\quad$ minutes & \\
Temperature during CPB & $29^{\circ} \mathrm{C} \pm 1^{\circ} \mathrm{C}$
\end{tabular}

Continuous variables are expressed as means \pm SD.

vivo neutrophil responsiveness to agonist stimulation during and after $\mathrm{CPB}$ was examined in an additional 8 patients. None of the patients had a history of chronic inflammatory disease, and all had stopped taking aspirin for a minimum of 5 days before surgery. All patients gave written informed consent for their participation in this study, which was approved by the Human Rights Committee of the University of Western Australia.

Operative technique. The same surgeon (M.N.) performed $\mathrm{CABG}$ using standard operative techniques and with antegrade and retrograde cold cardioplegic solution delivered through a catheter in the coronary sinus. All patients had an internal thoracic artery graft to the left anterior descending coronary artery, and most had multiple grafts (median, 3; range, 1-5). Oxygenation on bypass was through membrane diffusion, and no neutrophil filters were used. A bolus of heparin (3-4 mg/kg) was given after placement of aortic, atrial, and coronary sinus catheters, and further heparin was given during bypass to maintain an activated clotting time of greater than 300 seconds. The heparin was reversed with protamine sulfate after removal of lines. The mean aortic crossclamp and CPB times are shown in Table I.

Blood sample collections. At various times, $5 \mathrm{~mL}$ of blood was collected from the peripheral circulation (internal jugular vein), the ascending aorta, the coronary sinus, and the right and left atria. Blood taken before systemic heparinization was collected into heparinized tubes $(50 \mathrm{U} / \mathrm{mL})$. All other samples were collected in sterile polypropylene tubes without additives. Sampling was performed before anesthesia (T0), after sternotomy and before commencement of CPB (T1), during bypass just before release of the aortic crossclamp (T2), 5 minutes after removal of the aortic crossclamp (T3), just before the end of the bypass (mean $17 \pm 2$ minutes after removal of crossclamp; T4), and 5 minutes (T5) and 20 minutes (T6) after the bypass.

To assess neutrophil activation and sequestration in the left coronary circulation, aortic and coronary sinus blood was collected simultaneously before commencement of $\mathrm{CPB}$ (T1), 5 minutes after removal of the crossclamp (T3), just before the end of the bypass (T4), and at 5 minutes (T5) and 20 minutes (T6) after the bypass. The pulmonary circulation was similarly assessed by simultaneous sampling from the right and left atrium before commencement of CPB (T1) and at 5 minutes (T5) and 20 minutes (T6) after the bypass. 


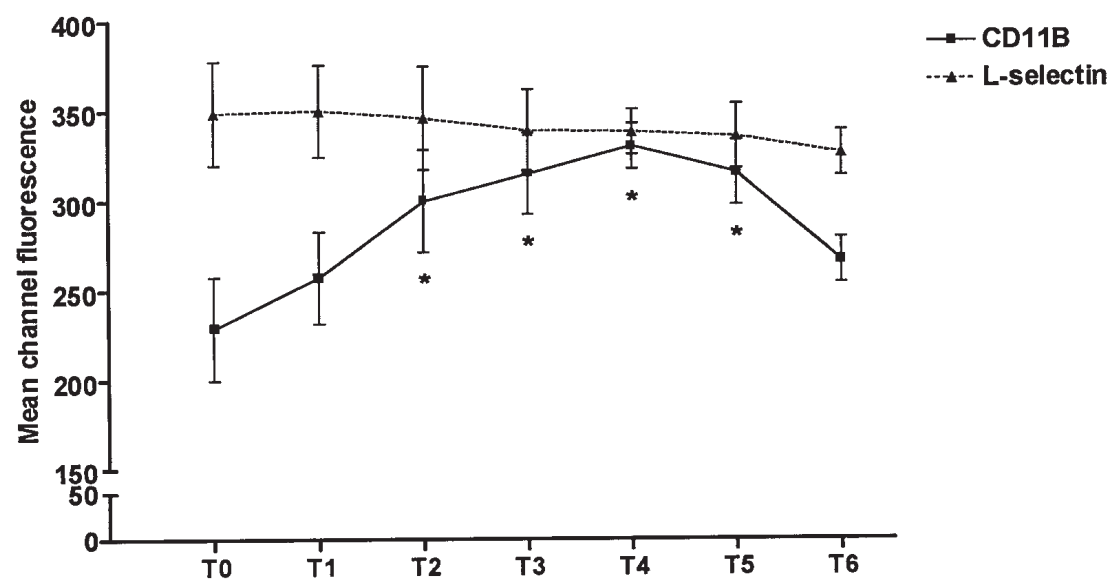

Fig 1. Peripheral blood neutrophil CD11b and L-selectin mean channel fluorescence (means \pm SEM) at preoperative baseline (T0), after sternotomy and before start of CPB (T1), before release of aortic crossclamp (T2), 5 minutes after removal of aortic crossclamp (T3), immediately before end of bypass (T4), and 5 minutes (T5) and 20 minutes $(T 6)$ after bypass $(\mathrm{n}=12)$. There was a significant increase in CD11b expression during bypass (ANOVA, $P=.003)$ but no change in L-selectin expression $(P>.2) .{ }^{*} P<.05$ versus preoperative study (T0).

Cell counting. Neutrophil counts and hematocrit levels were determined on a Coulter Cell counter (Department of Haematology, PathCentre, QEII Medical Centre, Perth). The standard error for the leukocyte counts is reported by the manufacturer as less than $0.02 \times 10^{9}$ cells $/ \mathrm{L}$ (coefficient of variability, $<1.7 \% / 10,000$ cells). To correct for the effect of hemodilution during $\mathrm{CPB}$, the neutrophil counts were standardized against the baseline (preanesthetic) hematocrit level by means of the following equation: corrected neutrophil count $=$ observed neutrophil count $\times$ baseline hematocrit $/$ observed hematocrit of blood sample.

Neutrophil activation by flow cytometry. Neutrophil adhesion molecule expression was determined by indirect immunofluorescent staining of whole blood and flow cytometry. ${ }^{21}$ Duplicate aliquots of whole blood $(80 \mu \mathrm{L})$ were incubated in the dark at room temperature for 30 minutes, with 10 $\mu \mathrm{L}$ each of saturating concentrations of FITC- and PElabeled monoclonal antibodies. In each sample red blood cells were lysed, and leukocytes were fixed by incubation with $1.5 \mathrm{~mL}$ of FACS Lysing Solution (Becton Dickinson) for 10 minutes in the dark at room temperature. The samples were then centrifuged ( $300 \mathrm{~g}$ for 5 minutes), and the pellet was washed with phosphate-buffered saline (PBS) containing $0.1 \%$ azide and resuspended in $300 \mu \mathrm{L}$ of $0.5 \%$ ( vol $/ \mathrm{vol}$ ) formaldehyde in PBS. The samples were stored at $4{ }^{\circ} \mathrm{C}$ until analyzed later on the day of collection.

Neutrophil CD11b and L-selectin expression was measured on a FACScan flow cytometer (Becton Dickinson). The neutrophil population was identified on the basis of its characteristic forward and side angle light scatter, and exclusion of lymphocytes and monocytes from this gate was confirmed by specific CD45 and CD14 staining, with greater than 90\% neutrophils present in all cases. Five thousand events were acquired for each sample. Binding of the PE-labeled CD11b and FITC-labeled L-selectin antibodies to neutrophils was quantitated both as the percentage of neutrophils exhibiting specific PE or FITC fluorescence and the mean channel fluorescence (MCF) that exceeded nonspecific background fluorescence. The MCF is expressed in arbitrary units transformed to a linear scale from the $\log _{10}$ channel number of mean fluorescence and is directly proportional to the density of receptors expressed per cell.

Neutrophil responses to ex vivo stimulation. A second cohort of 8 patients was studied to determine whether peripheral blood neutrophil responsiveness to ex vivo agonist stimulation was altered during and after CPB. For the neutrophil stimulation assays, central venous blood samples were taken preoperatively, at 5 minutes after commencement of CPB, at 5 minutes after removal of the aortic crossclamp, and at 24 hours after CPB. Duplicate $80-\mu \mathrm{L}$ aliquots of whole blood were incubated with $\mathrm{C} 5 \mathrm{a}$ (1:100 and 1:10 dilution of zymosanactivated serum), $\mathrm{LTB}_{4}\left(10^{-8}\right.$ or $\left.10^{-6} \mathrm{~mol} / \mathrm{L}\right)$, or control (PBS) for 10 minutes at room temperature. The higher agonist concentrations were selected on the basis of previous work in our laboratory that showed that they produced near maximal expression of neutrophil surface markers in vitro (data not shown). Each sample was then labeled with PE-labeled CD11b and FITC-labeled L-selectin monoclonal antibodies for subsequent FACScan analysis, as detailed above. Neutrophil responses to agonist stimulation were determined by the change in MCF of neutrophils expressing CD11b and L-selectin.

Data processing and statistical analysis. Data are expressed as means \pm SEM unless otherwise stated. For multiple comparisons, repeated-measures analysis of variance (ANOVA) for matched samples was used, and where a significant difference was identified, pairwise comparisons were made by using the Dunnett's multiple comparison posttest (Instat 2 software; GraphPad, San Diego, Calif). 


\section{$\mathrm{CD} 11 \mathrm{~b}$}

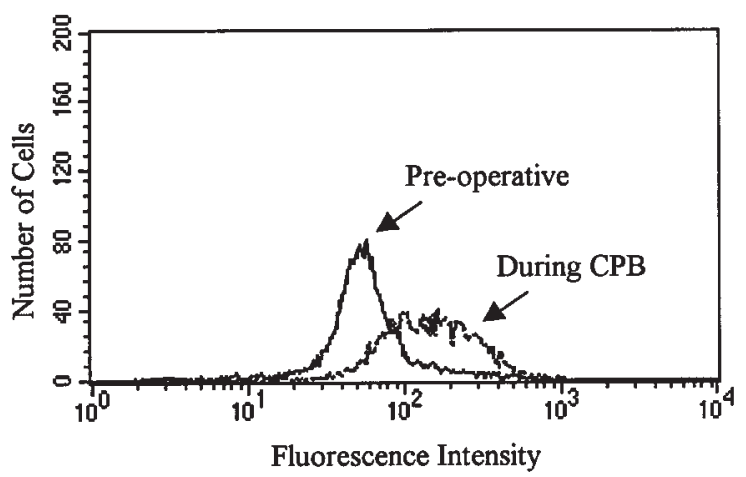

\section{L-selectin}

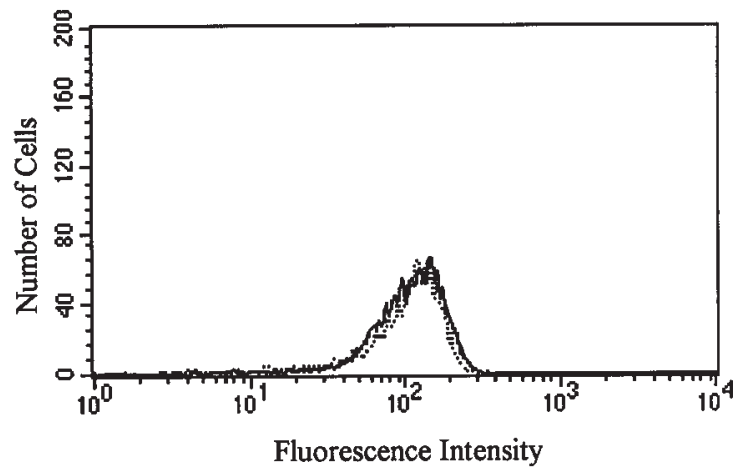

Fig 2. Flow cytometry histograms from one subject showing the fluorescence intensity on peripheral blood neutrophils preoperatively (solid line) and during CPB (broken line) when labeled for CD11b (top) and L-selectin (bottom). Note the increased fluorescence intensity of CD11b but the lack of change in fluorescence intensity of L-selectin on neutrophils sampled during CPB.

\section{Results}

Peripheral neutrophil count. At baseline, the neutrophil count in the peripheral blood was $3.3 \pm 0.5 \times$ $10^{9} / \mathrm{L}$. The corrected neutrophil count fell nonsignificantly during $\mathrm{CPB}$, but after release of the aortic crossclamp, there was a progressive increase in the peripheral neutrophil count to $10.4 \pm 1.5 \times 10^{9} / \mathrm{L}$ at 20 minutes after CPB (ANOVA, $P<.001$ ).

Peripheral blood neutrophil activation. Fig 1 shows MCF of CD11b and L-selectin on neutrophils in the peripheral circulation before, during, and after $\mathrm{CPB}$ (T0 to T6) in patients undergoing CABG $(n=12)$. Fig 2 shows representative flow cytometry histograms from one subject comparing fluorescence intensity for $\mathrm{CD} 11 \mathrm{~b}$ and L-selectin on circulating neutrophils preoperatively and during CPB. Over 90\% of the entire population of neutrophils expressed CD11b at preoperative baseline, and the percentage did not change throughout the time course of study. However, after the start of $\mathrm{CPB}$, there was a progressive upregulation of $\mathrm{CD} 11 \mathrm{~b}$ $\mathrm{MCF}$, indicating a significant increase in CD11b expression per cell, and this peaked after release of the aortic crossclamp, at which point it was $145 \%$ of baseline level (ANOVA, $P=.003$; Fig 1 ). After weaning off $\mathrm{CPB}$, the $\mathrm{CD} 11 \mathrm{~b} \mathrm{MCF}$ declined to a nonsignificant level above baseline at 20 minutes after bypass. Lselectin was also expressed on over $90 \%$ of the entire population of neutrophils at baseline, and we observed no downregulation (loss) of L-selectin expression either in the percentage positive cells or MCF throughout the course of the study (ANOVA, $P>.2$; Figs 1 and 2).

Neutrophil responsiveness to ex vivo agonist stimulation. Fig 3 shows the basal and ex vivo stimulated MCF of CD11b and L-selectin on peripheral blood neutrophils sampled at baseline, at 5 minutes after the onset of CPB, at 5 minutes after release of the aortic crossclamp, and at 24 hours after CABG $(n=8)$. Neutrophil responses to the high-agonist concentrations of $\mathrm{C} 5 \mathrm{a}$ (1:10 dilution of activated serum) and $\mathrm{LTB}_{4}\left(10^{-6} \mathrm{~mol} / \mathrm{L}\right)$ are shown. Neutrophil responses to C5a (1:100 dilution) and $\mathrm{LTB}_{4}\left(10^{-8}\right)$ were generally small (data not shown). As in the initial series, we found that the basal level of neutrophil CD11b MCF increased during $\mathrm{CPB}$, peaking after release of the aor- 

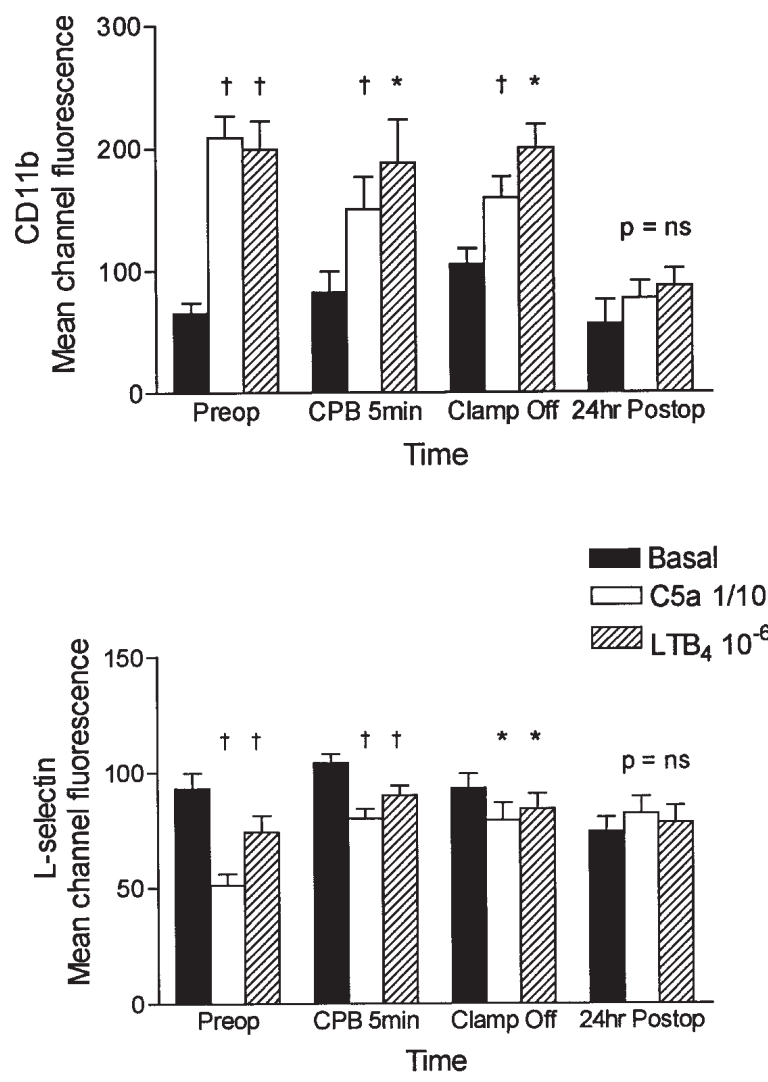

Fig 3. Bar graphs showing the basal and ex vivo stimulated mean channel fluorescence of CD11b (A) and L-selectin (B) on peripheral blood neutrophils sampled preoperatively (Preop), at 5 minutes after the onset of CPB (CPB $5 \mathrm{~min})$, at 5 minutes after release of aortic crossclamp (Clamp Off), and at 24 hours postoperatively $(24 h r$ Postop) $(\mathrm{n}=8)$. Neutrophil agonists were complement C5a (1:10 dilution of activated serum $)$ and $\operatorname{LTB}_{4}\left(10^{-6} \mathrm{~mol} / \mathrm{L}\right)$. Values are expressed as means \pm SEM. $* P<.01$ and $\dagger P<.001$ stimulated versus basal mean fluorescence expression of CD11b or L-selectin.

tic crossclamp to $161 \%$ of baseline level (ANOVA, $P<$ .001) but with no change in L-selectin expression. The $\mathrm{CD} 11 \mathrm{~b}$ and L-selectin MCF was not significantly different from the baseline level at 24 hours after CPB. At baseline, ex vivo stimulation by $\mathrm{C} 5 \mathrm{a}$ and $\mathrm{LTB}_{4}$ increased neutrophil CD11b MCF expression by more than 3-fold (both $P<.001$ ). There was still a significant response to ex vivo agonist stimulation by neutrophils sampled during $\mathrm{CPB}$, although there was a loss of response by neutrophils sampled 24 hours after CPB (Fig 3, A). A similar finding was observed with respect to L-selectin, where neutrophils sampled at baseline and during CPB responded to ex vivo agonist stimulation by a downregulation of L-selectin MCF $(P<.001)$, but neutrophils sampled at 24 hours after the operation failed to respond to stimulation (Fig $3, B$ ).

Neutrophil activation in coronary and pulmonary circulation. No significant changes in neutrophil $\mathrm{CD} 11 \mathrm{~b}$ or L-selectin expression, either percentage of positive cells or MCF, occurred on single pass across the left coronary circulation after release of the aortic crossclamp and at 5 and 20 minutes after CPB nor across the pulmonary circulation at 5 and 20 minutes after CPB (data not shown). However, comparison of the neutrophil counts in blood taken across the left coronary circulation after aortic crossclamp release demonstrated a $13 \%$ reduction in count after single pass, and this persisted to 20 minutes after patients were weaned off CPB (ANOVA, $P=.002$; Fig $4, A$ ). An $11 \%$ single-pass reduction in neutrophil count was also found across the pulmonary circulation at $20 \mathrm{~min}$ utes after CPB (ANOVA, $P=.02$; Fig $4, B$ ).

\section{Discussion}

This study was performed to provide a detailed profile of systemic and local neutrophil activation and adhesion molecule expression, as well as neutrophil responses to humoral stimulation in human subjects undergoing elective $\mathrm{CABG}$ with $\mathrm{CPB}$. The major findings were that (1) CPB was associated with a differential activation of adhesion molecules on circulating neutrophils with increased $\beta_{2}$-integrin (CD11b) but no significant change in L-selectin expression; (2) neutrophil CD11b expression peaked toward the end of $\mathrm{CPB}$ after release of the aortic crossclamp but declined rapidly after the end of $\mathrm{CPB}$; (3) there was a downregulation and loss of neutrophil responses to ex vivo agonist stimulation 24 hours after the operation; and (4) a significant loss of neutrophils, suggesting sequestration but no local neutrophil activation, could be demonstrated in the heart and lungs after reperfusion.

Similar to previous studies, we observed an initial mild neutropenia during CPB but a marked neutrophil mobilization and leukocytosis after CPB..$^{9,22}$ Neutrophil mobilization after $\mathrm{CPB}$ may be a response to the release of cytokines and inflammatory mediators from the heart and other organs on reperfusion, as suggested by previous studies. ${ }^{10-12}$ However, other factors, such as activation of complement by protamine administered at the end of surgery and circulatory rewarming, may stimulate neutrophil mobilization postoperatively., 9,22

Neutrophil activation with expression of surface adhesion molecules and the subsequent adhesion of neutrophils to the vascular endothelium appear to be key events initiating the inflammatory organ dysfunction after $\mathrm{CPB}$ and ischemia-reperfusion. ${ }^{2-6}$ Among the 

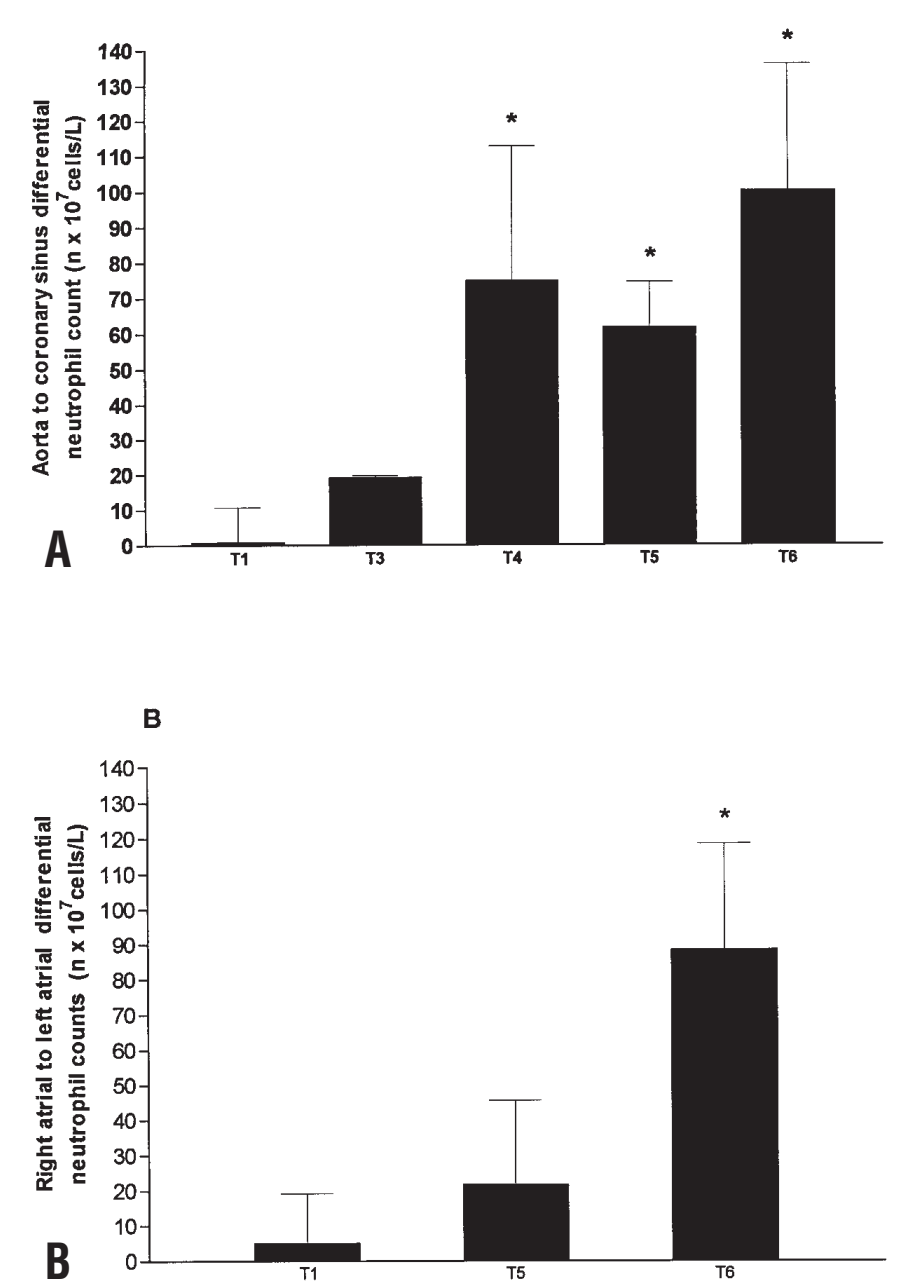

Fig 4. The differential in neutrophil count $\left(\mathrm{n} \times 10^{7} / \mathrm{L}\right)$ on single pass across the left coronary circulation $(\mathbf{A})$ and the pulmonary circulation (B) studied in 12 subjects before commencement of CPB (T1), 5 minutes after release of aortic clamp (T3), just before end of bypass (T4), and 5 minutes (T5) and 20 minutes after bypass (T6). Values expressed are means \pm SEM. There was a significant increase in differential neutrophil count across the left coronary circulation (ANOVA, $P=.002$ ), as well as the pulmonary circulation (ANOVA, $P=.02$ ), during postbypass reperfusion. ${ }^{*} P<.05$ differential neutrophil count at various time points versus baseline (T1).

leukocyte adhesion molecules studied so far, the $\beta_{2}$ integrins appear to be particularly important because blockade of the CD11/CD18 complex has been shown to effectively inhibit neutrophil influx and blunt tissue injury in many inflammatory conditions, including ischemia-reperfusion, ${ }^{5,6}$ the period after $\mathrm{CPB},{ }^{6,7}$ sepsis, ${ }^{23}$ and shock. ${ }^{24}$ Our finding that $\mathrm{CD} 11 \mathrm{~b}$ expression on circulating neutrophils was markedly upregulated during human $\mathrm{CPB}$ is therefore important and also consistent with that reported by other studies. ${ }^{14-16}$ In our 2 series of patients undergoing CABG, we found a consistent profile of neutrophil activation with a progressive upregulation of neutrophil CD11b expression after the onset of $\mathrm{CPB}$, which peaked after the release of the aortic crossclamp. The early upregulation of $\mathrm{CD} 11 \mathrm{~b}$ expression during CPB is most likely related to complement activation from blood-material contact in the extracorporeal circuit. ${ }^{8,9}$ However, the late peaking of neutrophil CD11b expression after aortic crossclamp release suggests an additional activation of neutrophils related to coronary reperfusion. Our finding is supported by $\mathrm{Gu}$ and colleagues, ${ }^{25}$ who also reported that release of the aortic crossclamp induced a second peak of leukocyte complement-receptor type 3 (mac-1) expression, paralleled by an increase in $\mathrm{LTB}_{4}$ secretion in the late period of $\mathrm{CPB}$. We found that $\mathrm{CD} 11 \mathrm{~b}$ 
expression on circulating neutrophils declined rapidly after bypass, but variables such as the length of CPB and normothermic versus hypothermic cardioplegia may explain observed differences in the extent of continuing neutrophil activation postoperatively. ${ }^{14-16}$ Our subsequent data also suggest that the early decline of CD11b expression may be due to a desensitization of neutrophils to humoral stimulation.

Unlike CD11b, we found no significant activation (shedding) of L-selectin on circulating neutrophils. This may appear surprising given that L-selectin is important in the initial margination of leukocytes from the normal blood flow, but other studies have also failed to show activation of L-selectin on neutrophils in the peripheral circulation during CPB. ${ }^{15,19,20}$ By contrast, Finn and colleagues ${ }^{17}$ and Moen and colleagues ${ }^{18}$ reported a significant downregulation of L-selectin on neutrophils during human $\mathrm{CPB}$, but this was restricted to a small proportion of cells, unlike CD11b expression, which was uniformly increased in the entire neutrophil population. Thus we may have failed to detect a change in L-selectin expression on circulating neutrophils because it was confined to a small proportion of activated neutrophils that were then trapped in the microcirculation. However, a more likely interpretation is that the generalized inflammation associated with $\mathrm{CPB}$ can upregulate neutrophil $\beta_{2}$-integrins without involvement of L-selectin. ${ }^{26}$ The fact that most patients recover uneventfully after cardiac operations despite the generalized neutrophil activation suggests that other mechanisms exist in vivo to limit the inflammatory response. Indeed, one of the most interesting observations in our study was that circulating neutrophils appeared to become anergic to ex vivo agonist stimulation with $\mathrm{C} 5 \mathrm{a}$ and $\mathrm{LTB}_{4}$ by 24 hours postoperatively. There are also reports that neutrophil responses to stimulation by complement or IL-8 may be reduced after CPB. ${ }^{27,28}$ These results suggest that being able to pharmacologically downregulate the response by neutrophils, for example by use of $\mathrm{LTB}_{4}$ receptor antagonists, may be of benefit in patients undergoing CPB.

We found that there was a significant loss of neutrophils across the coronary and pulmonary circulations after bypass, suggesting that neutrophils were being sequestered. Other studies have also suggested that neutrophil sequestration occurs in the heart during reperfusion after bypass, ${ }^{29,30}$ as well as in the lungs, where it has been correlated with postbypass ventilatory impairment. ${ }^{8,9}$ Despite the evident neutrophil sequestration, we were unable to show a differential of CD11b or L-selectin expression on neutrophils crossing both vascular beds during reperfusion. This does not entirely exclude local neutrophil activation because it would have been difficult to detect a small change in $\mathrm{CD} 11 \mathrm{~b}$ expression during a single pass across the vascular bed when it was already highly expressed on the majority of circulating neutrophils. Nevertheless, the upregulated $\mathrm{CD} 11 \mathrm{~b}$ expression on trafficking neutrophils is likely to contribute to neutrophil adhesion and vascular transmigration. The fact that significant neutrophil disappearance in the heart and lungs was not found until some 15 to 20 minutes after reperfusion suggests that there are time-dependent local factors determining neutrophil adhesion and transmigration. This may include a progressive recruitment of local inflammatory responses in the microcirculation after ischemic reperfusion and expression of counter ligands for neutrophils on activated endothelial cells. It is also possible that neutrophils were later trapped by microvascular plugging.

The clinical implications of our study are uncertain because we did not correlate the findings with postbypass cardiac or ventilatory function in our patients. However, they were unlikely to have clinically apparent impairment because of good left ventricular function and short CPB times. Nevertheless, the demonstration that even in this group of patients there was evidence of a marked neutrophil activation and subsequent neutrophil sequestration in the heart and lungs suggests a need for further studies in patients with unstable syndromes, active ischemia, or long CPB times undergoing CABG. Furthermore, this study emphasizes the potential of targeting leukocyte CD11b-CD18 expression, either by preventing its activation or by its blockade, as a strategy to reduce the inflammatory organ dysfunction associated with cardiopulmonary bypass.

We thank the Departments of Haematology and Anaesthesiology, Sir Charles Gairdner Hospital, for providing valuable technical assistance.

\section{REFERENCES}

1. Kirklin JK, McGiffin DC. Early complications following cardiac surgery. Cardiovasc Clin 1987;17:321-42.

2. Elliot MJ, Finn AHR. Interaction between neutrophils and endothelium. Ann Thorac Surg 1993;56:1503-8.

3. Shappell SB, Toman C, Anderson DC, Taylor AA, Entman ML, Smith CW. Mac-1 (CD11b/CD18) mediates adherence-dependent hydrogen peroxide production by human and canine neutrophils. J Immunol 1990;56:1503-8.

4. Bando K, Pillai R, Cameron DE, Brawn JD, Winkelstein JA, Hutchins GM, et al. Leukocyte depletion ameliorates free radicalmediated lung injury after cardiopulmonary bypass. J Thorac Cardiovasc Surg 1990;99:873-7.

5. Simpson PJ, Todd RFI, Fantone JC, Mickelson JK, Griffin JD, 
Luchessi BR. Reduction of experimental canine myocardial reperfusion injury by a monoclonal antibody (anti-Mo1, and antiCD11b) that inhibits leukocyte adhesion. J Clin Invest 1988;81: 624-9.

6. Verrier ED, Shen I. Potential role of neutrophil anti-adhesion therapy in myocardial stunning, myocardial infarction, and organ dysfunction after cardiopulmonary bypass. J Card Surg 1993;8: 309-12.

7. Dreyer WJ, Michael LH, Millman EE, Berens KL, Geske RS. Neutrophil sequestration and pulmonary dysfunction in a canine model of open heart surgery with cardiopulmonary bypass: evidence for a CD18-dependent mechanism. Circulation 1995;92: 2276-83.

8. Cavarocchi NC, England MD, Schaff HV, Russo P, Orszulak TA, Schnell WA, et al. Oxygen free radical generation during cardiopulmonary bypass: correlation with complement activation. Circulation 1986;74(Suppl):III130-3.

9. Howard RJ, Crain C, Franzini DA, Hood I, Hugli TE. Effect of cardiopulmonary bypass on pulmonary leukostasis and complement activation. Arch Surg 1988;123:1496-501.

10. Dauber IM, Parsons PE, Welsh CH, Giclas PC, Whitman GJR, Wheeler GS, et al. Peripheral bypass-induced pulmonary and coronary vascular injury: association with increased levels of tumor necrosis factor. Circulation 1993;88:726-35.

11. Frering B, Philip I, Dehoux M, Rolland C, Langlois JM, Desmonts JM. Circulating cytokines in patients undergoing normothermic cardiopulmonary bypass. J Thorac Cardiovasc Surg 1994;108:636-41.

12. Gadaleta D, Fahey AL, Verma M, Ko W, Kreiger KH, Isom OW, et al. Neutrophil leukotriene generation increases after cardiopulmonary bypass. J Thorac Cardiovasc Surg 1994;108:642-7.

13. Adams DH, Shaw S. Leucocyte-endothelial interactions and regulation of leucocyte migration. Lancet 1994;343:831-6.

14. Gillinov AM, Bator JM, Zehr KJ, Redmond JM, Burch RM, Ko $\mathrm{C}$, et al. Neutrophil adhesion molecule expression during cardiopulmonary bypass with bubble and membrane oxygenators. Ann Thorac Surg 1993;56:847-53.

15. Le Deist F, Menasche P, Kucharski C, Bel A, Piwnica A, Bloch G. Hypothermia during cardiopulmonary bypass delays but does not prevent neutrophil-endothelial cell adhesion: a clinical study. Circulation 1995;92(Suppl):II354-8.

16. Takala AJ, Jousela IT, Takkunen OS, Jansson SE, Kyosola KT, Olkkola KT, et al. Time course of beta 2-integrin CD11b/CD18 (Mac-1, alpha M beta 2) upregulation on neutrophils and monocytes after coronary artery bypass grafting: CD11b upregulation after CABG surgery. Scand J Thorac Cardiovasc Surg 1996;30: 141-8.

17. Finn A, Moat N, Rebuck N, Klein N, Strobel S, Elliott M. Changes in neutrophil CD11b/CD18 and L-selectin expression and release of interleukin 8 and elastase in paediatric cardiopulmonary bypass. Agents Actions 1993;38:C44-6.
18. Moen O, Hogasen K, Fosse E, Dregelid E, Brockmeier V, Venge $\mathrm{P}$, et al. Attenuation of changes in leukocyte surface markers and complement activation with heparin-coated cardiopulmonary bypass. Ann Thorac Surg 1997;63:105-11.

19. Le Deist F, Menasche P, Bel A, Lariviere J, Piwnica A, Bloch G. Patterns of changes in neutrophil adhesion molecules during normothermic cardiopulmonary bypass: a clinical study. Eur J Cardiothorac Surg 1996;10:279-83.

20. Galiñanes M, Watson C, Trivedi U, Chambers DJ, Young CP, Venn GE. Differential patterns of neutrophil adhesion molecules during cardiopulmonary bypass in humans. Circulation 1996;94(Suppl):II364-9.

21. Ilton MK, Taylor M, Misso N, Thompson PJ, Hung J. Neutrophil cathepsin $\mathrm{G}$ modulates platelet P-selectin expression and inhibits P-selectin mediated platelet-neutrophil adhesion. Clin Sci 1998; 94:437-45.

22. Quiroga MM, Miyagishima R, Haendschen LC, Glovsky M, Martin BA, Hogg JC. The effect of body temperature on leukocyte kinetics during cardiopulmonary bypass. J Thorac Cardiovasc Surg 1985;90:91-6.

23. Walsh CJ, Carey PD, Cook DJ, Bechard DE, Fowler AA, Sugerman HJ. Anti-CD18 antibody attenuates neutropenia and alveolar capillary-membrane injury during gram-negative sepsis. Surgery 1991;110:205-11.

24. Vedder NB, Winn RK, Rice CL, Chi EY, Arfors KE, Harlan JM. A monoclonal antibody to adherence-promoting leukocyte glycoprotein, CD18, reduces organ injury and promotes survival from hemorrhagic shock and resuscitation in rabbits. J Clin Invest 1988;81:939-44

25. Gu YJ, van Oeveren W, Boonstra PW, de Haan J, Wildevuur CRH. Leukocyte activation with increased expression of CR3 receptors during cardiopulmonary bypass. Ann Thorac Surg 1992;53:839-43

26. Albelda SM, Smith CW, Ward PA. Adhesion molecules and inflammatory injury. FASEB J 1994;8:504-12.

27. Dreyer WJ, Smith W, Entman ML. Invited letter to the editor: Neutrophil activation during cardiopulmonary bypass. J Thorac Cardiovasc Surg 1991;102:318-20.

28. Ng TT, Gibson FA, Nye KE, Hughes PJ, Hinds CJ, Morrow WJ, et al. Desensitization of the inflammatory response in humans: changes in response to cardiopulmonary bypass. Shock 1997; 8:159-64.

29. Farah B, Vuillemenot A, Lecompte T, Bara L, Pasquier C, Jebara $\mathrm{V}$, et al. Myocardial neutrophil sequestration and activation related to reperfusion of human heart during coronary artery surgery. Cardiovasc Res 1994;28:1226-30.

30. Curello S, Ceconi C, de Giuli F, Panzali AF, Milanesi B, Calarco $\mathrm{M}$, et al. Oxidative stress during reperfusion of human hearts: potential sources of oxygen free radicals. Cardiovasc Res 1995; 29:118-25. 